\title{
Entrelacs
}

Cinéma et audiovisuel

\section{Le mécanisme du corps comme manifestation de l'essence pure chez Robert Bresson}

\section{Peggy Saule}

\section{(2) OpenEdition}

Journals

Édition électronique

URL : http://journals.openedition.org/entrelacs/64

DOI : $10.4000 /$ entrelacs.64

ISSN : 2261-5482

Éditeur

Éditions Téraèdre

\section{Édition imprimée}

Date de publication : 1 novembre 2005

Pagination : 73-77

ISBN : 978-2-912868-70-1

ISSN : 1266-7188

\section{Référence électronique}

Peggy Saule, «Le mécanisme du corps comme manifestation de l'essence pure chez Robert Bresson », Entrelacs [En ligne], 5 | 2005, mis en ligne le 01 août 2012, consulté le 01 mai 2019. URL http://journals.openedition.org/entrelacs/64; DOI : 10.4000/entrelacs.64

Ce document a été généré automatiquement le 1 mai 2019.

Tous droits réservés 


\title{
Le mécanisme du corps comme manifestation de l'essence pure chez Robert Bresson
}

\author{
Peggy Saule
}

1 La distanciation entre l'âme et le corps est une querelle incessante chez les philosophes. Depuis la théorie de la connaissance platonicienne, qui, à travers la réminiscence des Idées fait passer de la connaissance intelligible à l'expérience sensible, jusqu'à la tradition scolastique qui considère le corps comme une machine sophistiquée répondant aux intentions de l'âme, l'être est présenté comme un "automate spirituel $»^{1}$ en tant que l'âme et le corps sont distincts l'un de l'autre par la primauté et l'antériorité de l'esprit sur le corps. C'est notamment avec Spinoza que ces rapports évoluent : l'attribut Pensée et l'attribut Etendue sont parallèles entre eux - c'est-à-dire équivalents et simultanés.

2 Cependant, la pensée dont il est question est à différencier de la raison. En effet, la raison est une appropriation de l'être au travers d'une conceptualisation formelle dont la vérité tautologique fige tout contexte. Cette fixation théorique et temporelle suppose l'autoréflexion du discours. Alors que la pensée, qui est l'autre de la raison, ne se réfléchit pas et ne réfléchit pas. Elle est une construction permanente de l'esprit : la pensée se fait au présent et en fonction des événements qui surviennent, la pensée se fait en mouvement. Dès lors on peut dire que l'esprit raisonne et que le corps pense. En ce sens nous pouvons comprendre le traitement du corps chez Bresson car le cinéaste introduit l'idée de parfaite adéquation entre la pensée et le geste.

3 C'est ce « corps-pensant » qui est magnifié par le cinématographe ${ }^{2}$ de Bresson. Le corps n'est plus « acté » par l'âme car il a lui-même des intentions et des significations propres. Les actions ne sont plus télécommandées par les décisions de l'esprit; elles sont signe d'engagement et de révélation de l'être. Par ses gestes, l'individu exprime toute son essence, toute sa spécificité ontologique, ce qui le rend si singulier et unique.

Le « corps-pensant » de Bresson nous entraîne dans une virtuosité cinématographique où la composition fragmentaire des plans, l'automatisme gestuel et l'invariant intonatif reflètent la déflagration mentale des personnages. 


\section{La mécanique des corps}

La fascination du corps, chez Robert Bresson, est telle qu'il n'a de cesse de juger de son individualité propre, de sorte qu'il inaugure en chaque mouvement et en chaque membre une autonomie et une nécessité substantielle. Plutôt que de proposer une vision du corps dans son harmonieuse beauté, nous voyons dans ses films des corps découpés par une fragmentation obsessionnelle rendant à chaque partie son tout.

En effet, le dépouillement corporel est absolu : les personnages bressoniens sont des corps avant d'être des individus. Le corps est rarement filmé dans son ensemble, il est segmentarisé par des gros plans sur les yeux, le visage, les mains, les jambes, les pieds etc... Le corps, dénudé ou non, n'est visible que comme morceaux-de-corps. Chaque partie existe pour elle-même.

7 Ce plan du Procès de Jeanne D'Arc témoigne de la fragmentation esthétique: les extrémités du corps de Jeanne (Florence Delay) sont la représentation de sa situation de prisonnière (pieds attachés) et de son élan de révolte (main ramassant une pierre jetée par la foule).

La fragmentation est indispensable si on ne veut pas tomber dans la représentation. Voir les êtres et les choses dans leurs parties séparables. Isoler ces parties. Les rendre indépendantes afin de leur donner une nouvelle dépendance ${ }^{3}$.

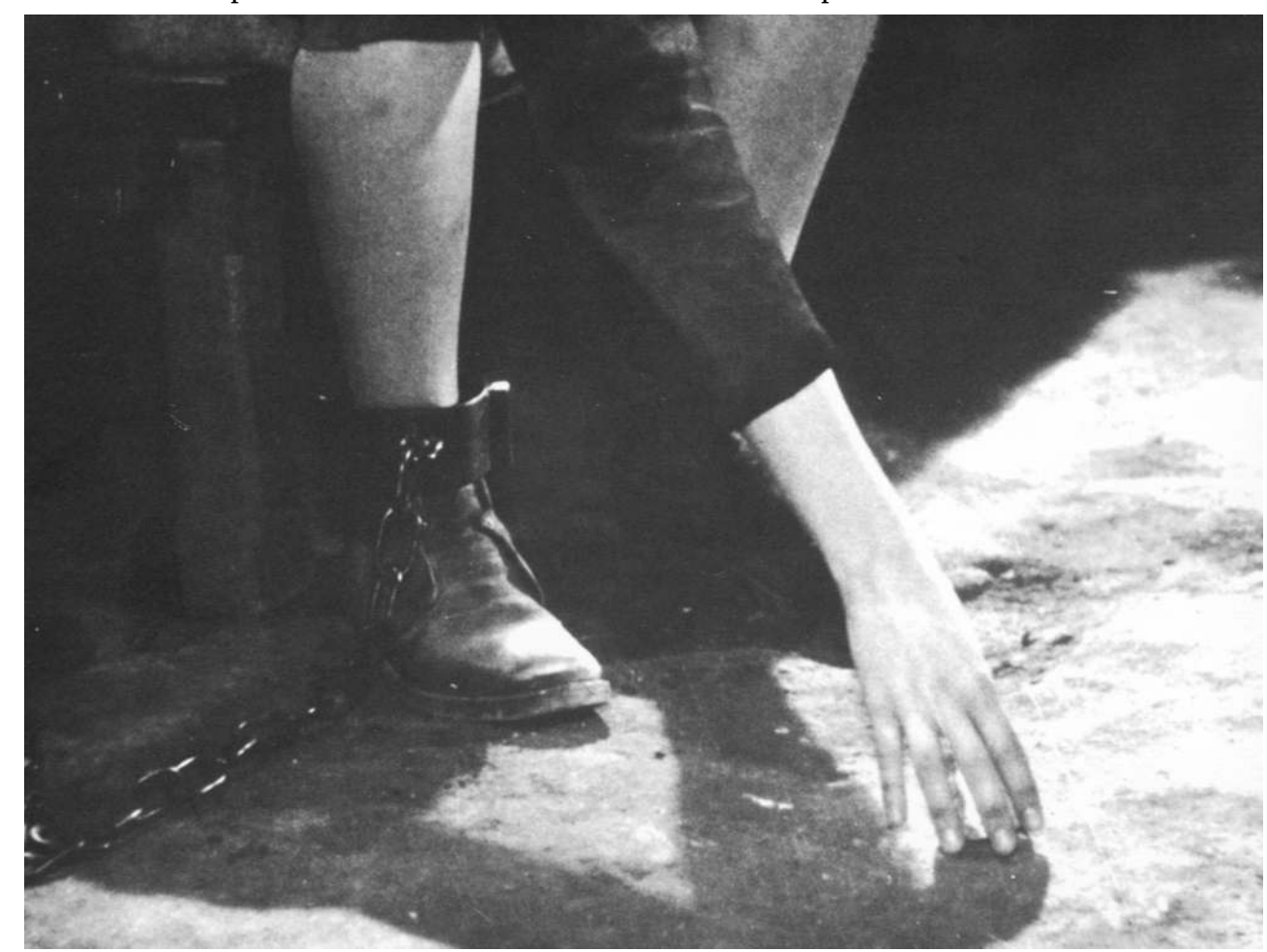




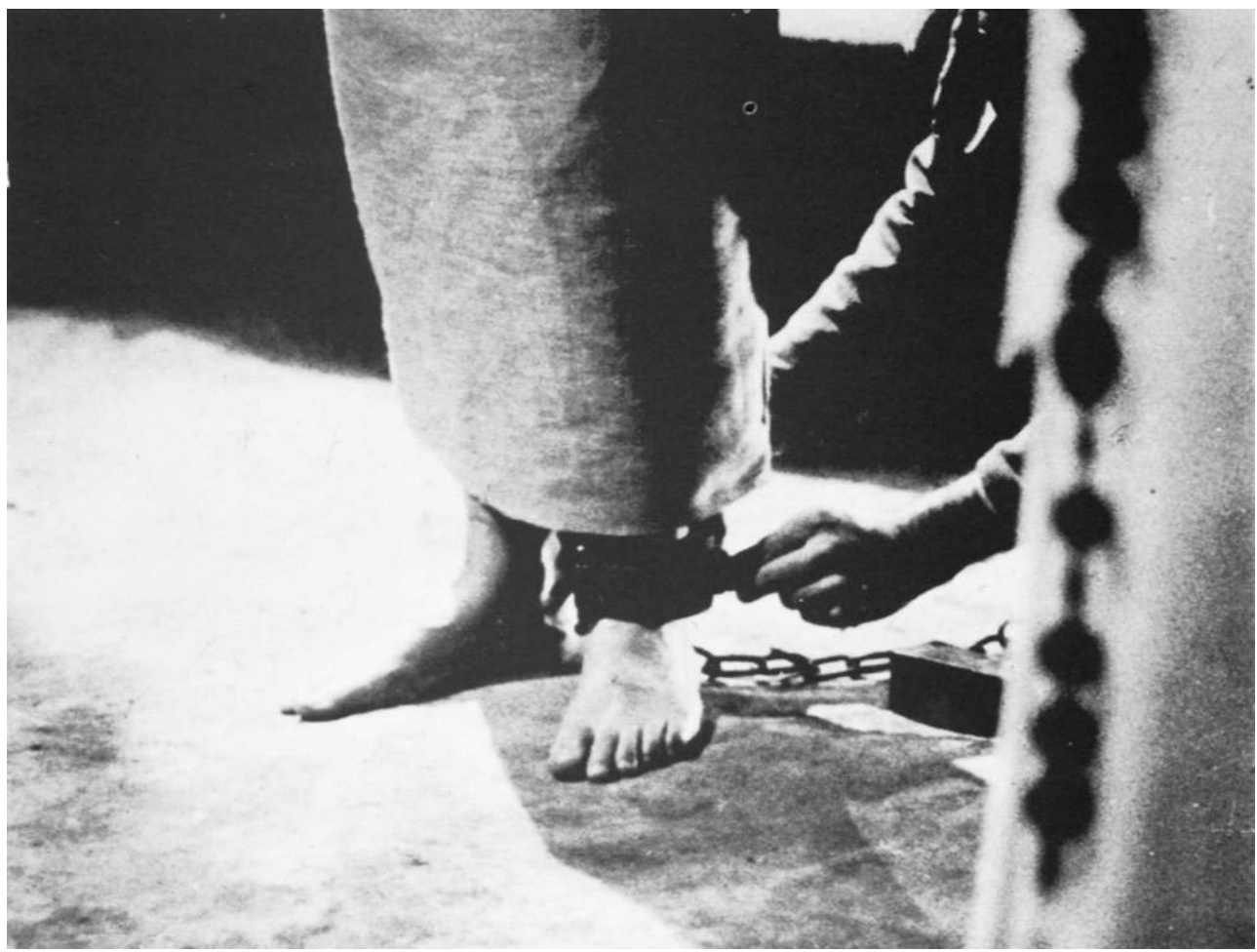

8 Les corps sont mémorables grâce à l'attribution d'une nouvelle cohérence: la fragmentation permet de décomposer le réel pour recomposer une réalité cinématographique. Il s'agit d'un découpage corporel donnant au corps la symbolique souhaitée: le corps mécanique. Si bien que le ballet bressonien consiste en une chorégraphie abstraite de membres détachés.

9 Nous rencontrons, dans ce plan de Lancelot du lac, un remarquable exemple du projet artistique de la fragmentation; la multiplicité des césures visuelles rend compte de l'intention créatrice du cinéaste. 


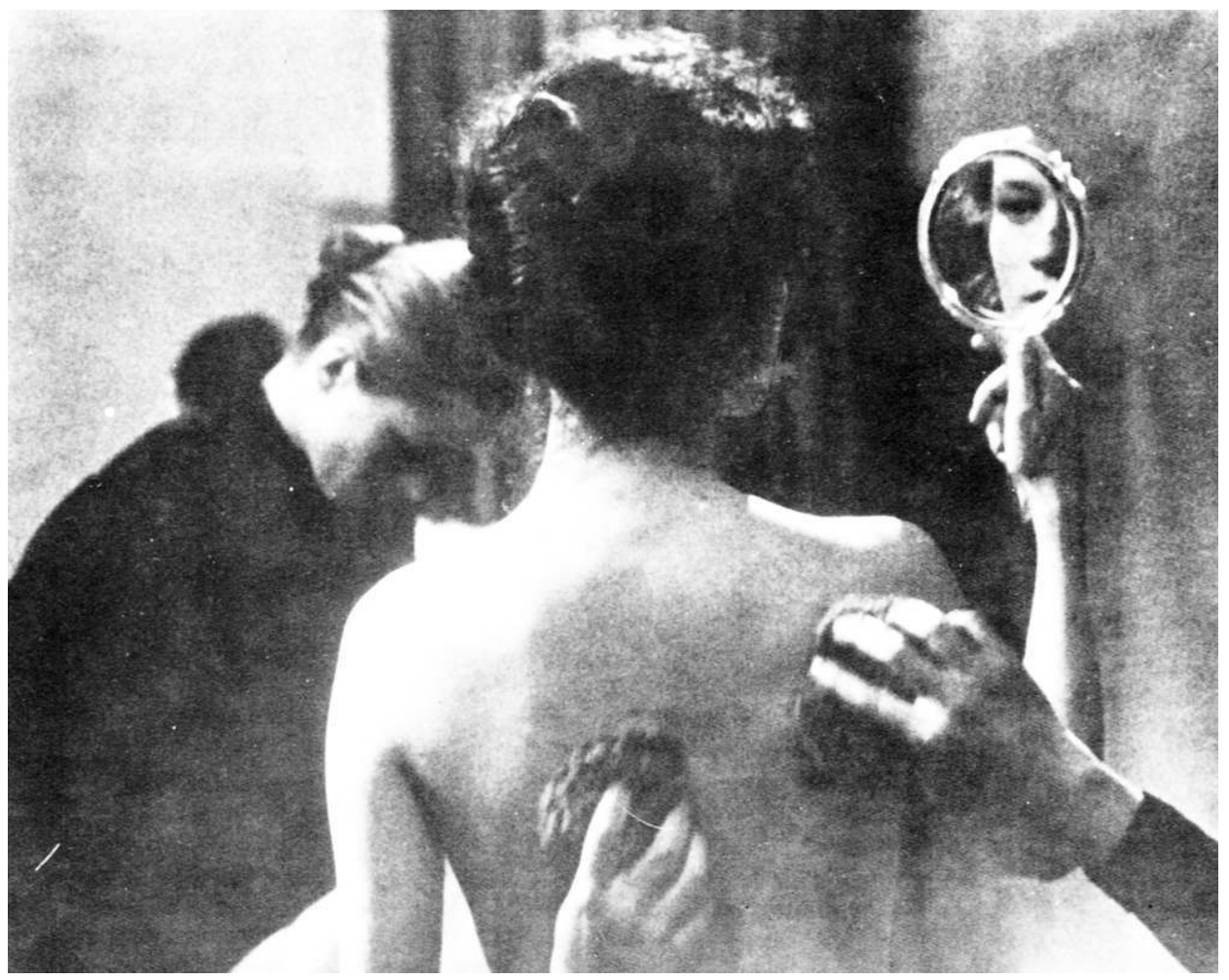

10 Au premier plan, le dos dénudé de la reine Guenièvre est voilé par deux mains ; la vision du bras de la jeune femme est empêchée; le reflet du miroir ne nous laisse entrevoir que la moitié de son visage.

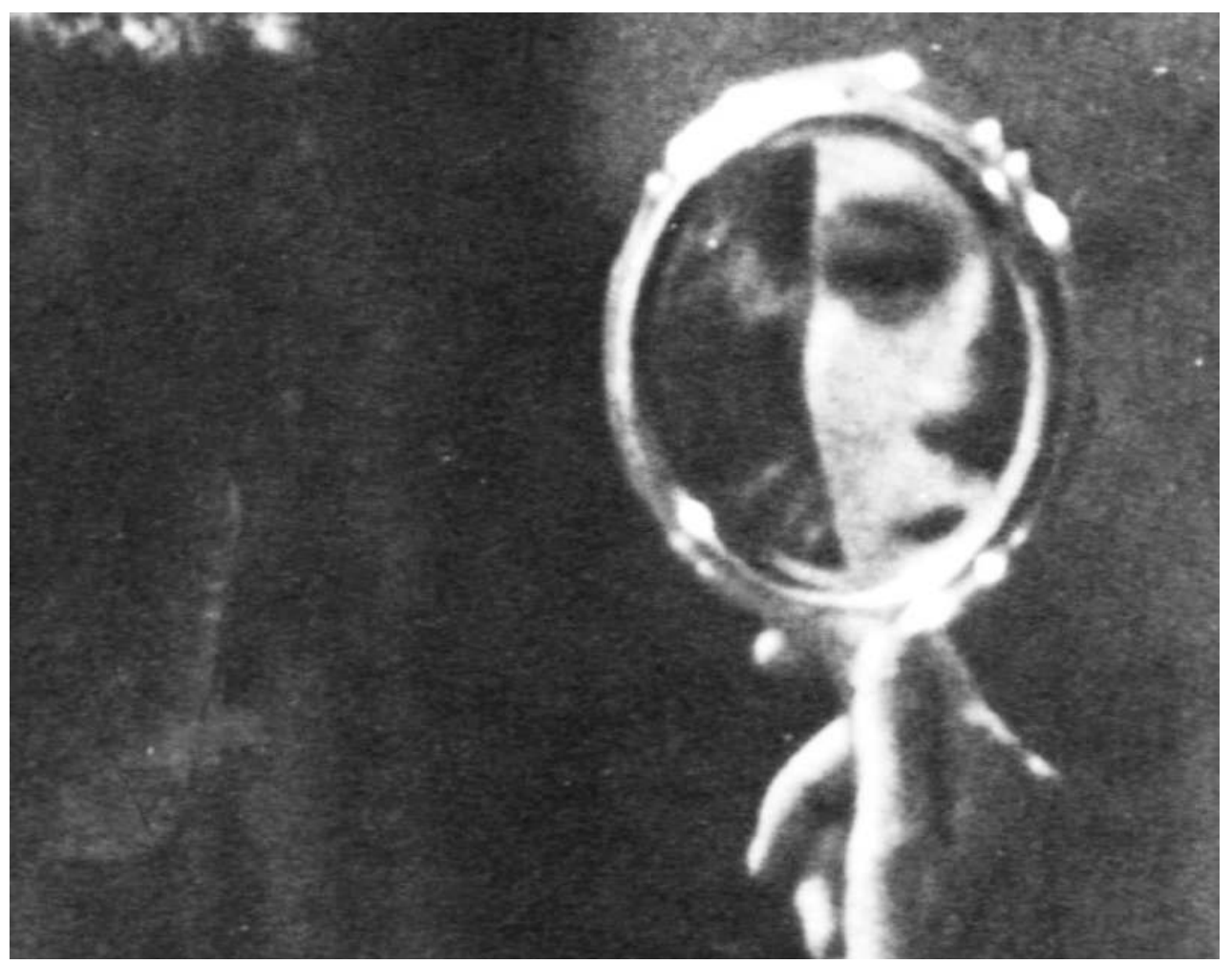

11 Au second plan, la tête courbée et de profil de la femme vêtue d'un manteau ne permet de distinguer que sa coiffure. 


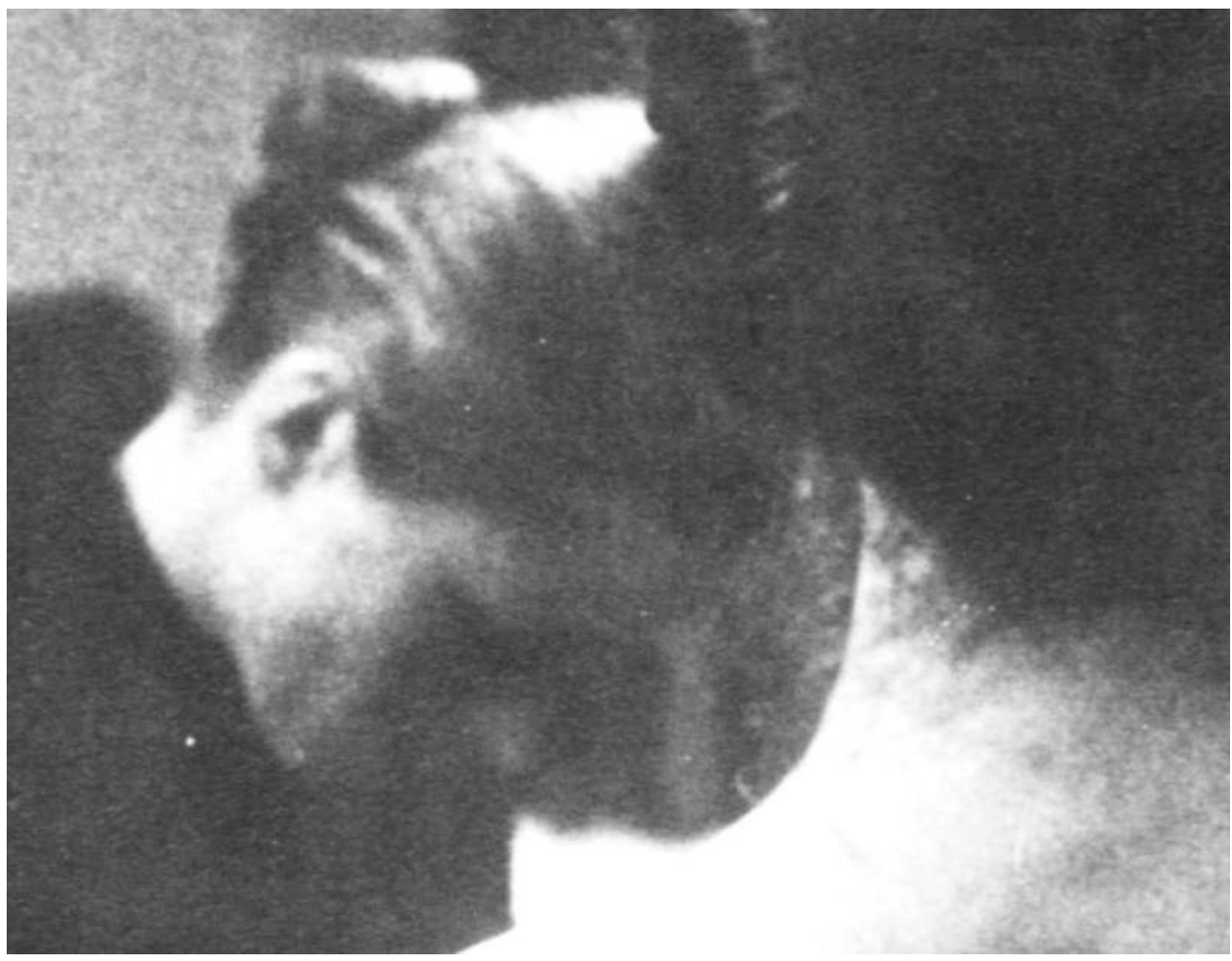

12 Interférences des perspectives et obstruction du champ visuel, tels sont les éléments de distorsion de la réalité. Aucun corps n'est vu dans son ensemble, cependant rien ne rompt la continuité picturale crée par le mouvement d'orientation vers la droite (trois mains droites, les deux têtes des femmes sont dirigées vers la droite, reflet de la moitié droite du visage dans le miroir). Cette image non cohésive et néanmoins cohérente ainsi que le jeu elliptique sur le « voir-non-vu» ajoute à la nudité fractionnée un regard presque érotique de la représentation féminine. La lumière blanche purifie le sujet: dès lors on s'aperçoit que l'œil du spectateur - attiré par l'œil de la jeune fille qui se mire - est davantage le témoin d'une contemplation qui suggère l'iconoclaste angélique.

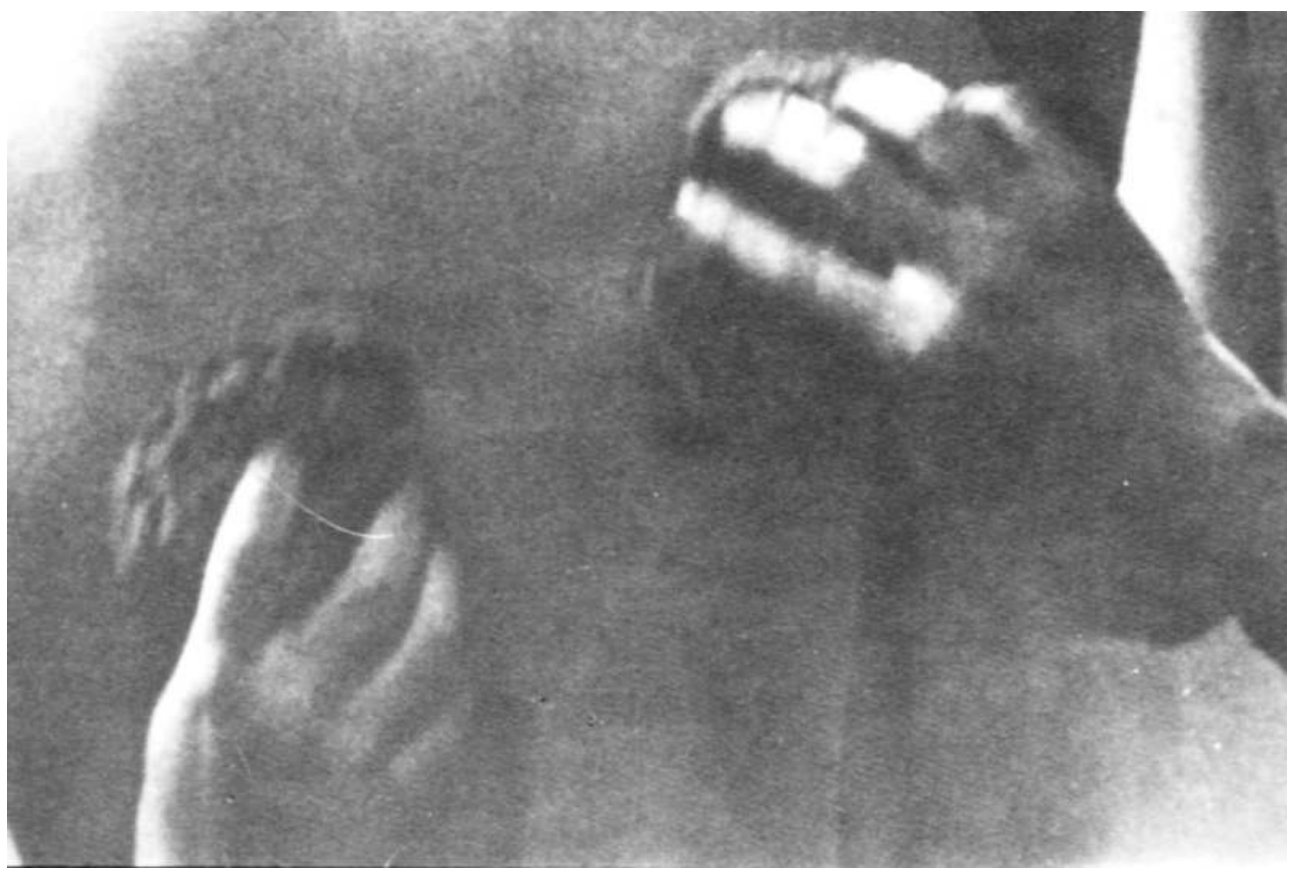


13 La dislocation plastique n'est pas la finalité du cinéaste qui ne cherche pas à montrer un monde divisé, mais à créer lui-même un univers morcelé, métaphore d'une réalité incompréhensible et désordonnée. Cet effet de composition est visible non seulement dans l'accumulation et l'alternance contrastée de gros plans et plans d'ensemble, mais aussi dans un montage brutal où des éléments de réel se juxtaposent et s'entrechoquent. L'espace visuel est un espace déconnecté dont les parties implorent au spectateur un raccordement immédiat de l'imagination. La volonté bressonienne est de rendre compte, au travers d'un jeu de déambulation des corps, de l'impuissance des individus pris dans l'engrenage d'une destinée qui leur échappe.

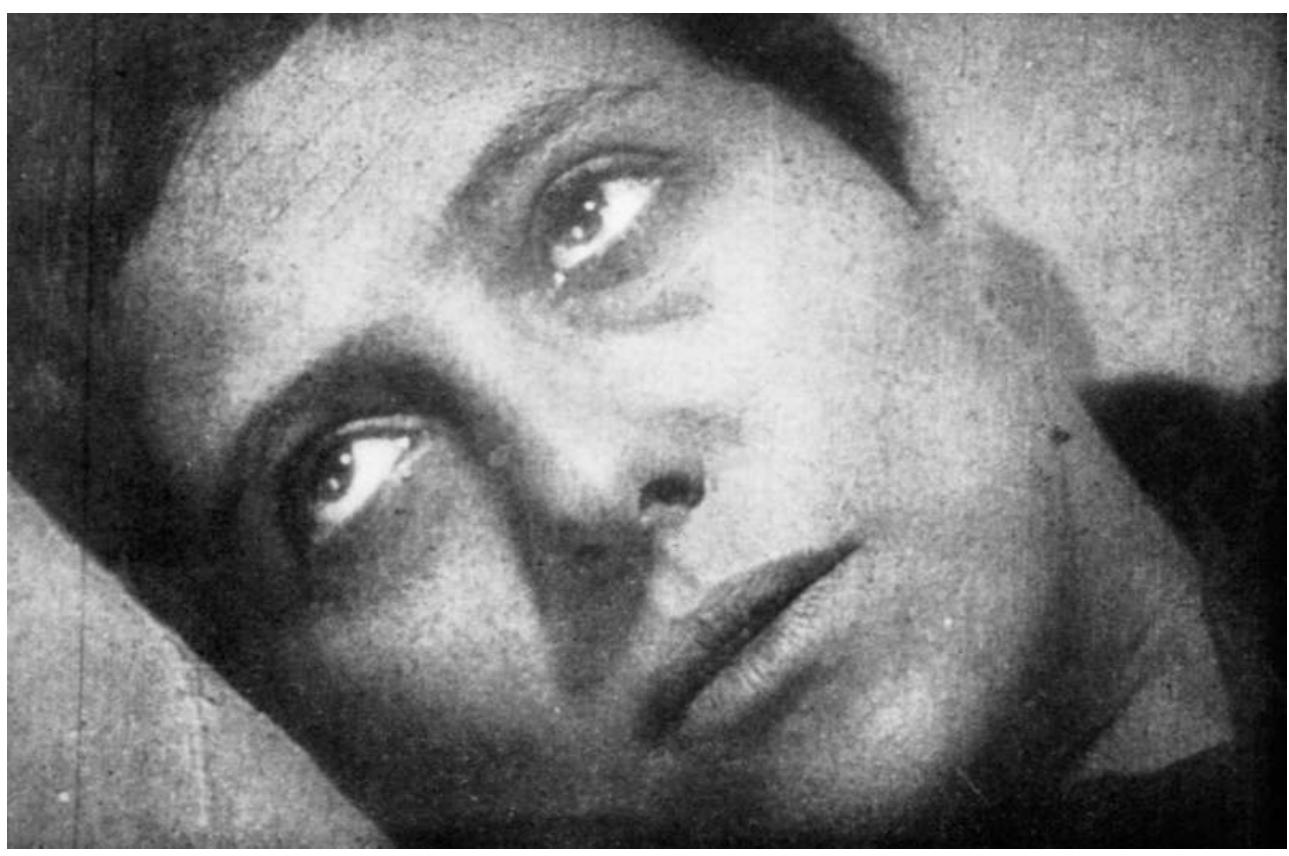

14 Avant le sacrifice ultime, la caméra bressonienne ne traque pas l'émotion de Jeanne D'Arc (comme a pu le faire Dreyer avec ses gros-plans) mais filme l'inéluctable destinée d'une jeune femme vaincue.

\section{Représentation de l'automate spirituel}

Par ailleurs, le cadrage de la caméra, est à la fois linéaire - peu de mouvements de plongée et contre-plongée - et réglé à la hauteur fixe, si bien que les têtes des adultes se trouvent souvent hors-cadre. L'image non conventionnelle de «corps-sans-têtes» nous montre à quel point le corps a en lui-même une indépendance spirituelle. Le corps n'a pas besoin d'être pensé pour agir car il peut agir selon sa propre entité individuelle. C'est un corps-pensant. Petit à petit, la construction de l'espace devient adéquate aux décisions de l'esprit. Ces corps infirmes ressemblent étrangement aux créatures monstrueuses d'Empédocle nées de l'assemblage d'éléments de corps épars, mais constitutives de l'étape préliminaire et nécessaire à la création de l'Humanité et de l'Ordre du monde. Pour Empédocle comme pour Bresson, c'est du monstrueux que naît l'harmonie, du fragmentaire que naît la cohésion.

16 Il s'agit pour Robert Bresson de stigmatiser le mécanisme interne du corps en laissant place à « un contrôle supérieur unissant la pensée critique et consciente à l'inconscient de la pensée : l'automate spirituel » ${ }^{4}$. Dès lors, «l'automate spirituel » prend un nouveau sens : il ne 
signifie plus la toute puissance de l'âme sur le corps et s'autorise avant tout à accorder au corps le pouvoir de découvrir l'âme du modèle 5 .

Ainsi l'automate ${ }^{6}$ signifie chez Bresson non seulement autonomie fragmentaire du contenu des images, mais aussi autonomie psychologique des personnages dans le sens où les modèles ne franchissent jamais le seuil de la zone étrangère du hors-moi. Chaque modèle est intrinsèquement enfermé en lui-même, inexorablement égocentré. Les relations inter-humaines ne participent pas à sa complétude atypique car les personnages se croisent mais ne se rencontrent pas, se parlent mais ne communiquent pas.

Enfin, l'automate suppose l'autonomie mécanique du geste.

Avec Bresson, c'est un troisième état qui apparaît où l'automate est pur, aussi privé d'idées que de sentiments, réduits à l'automatisme des gestes quotidiens segmentarisés, mais doués d'autonomie ${ }^{7}$.

19 En effet, pour accéder à l'automatisme spirituel, c'est-à-dire à ce rapport de réciprocité entre le mouvement automatique et l'automatisme intellectuel est nécessaire le procédé de réitération chronique et monotone des mouvements. C'est pourquoi Bresson impose à ses acteurs un très grand nombre de prises pour chaque scène afin que les gestes deviennent automatiques, presque instinctifs car plus un geste est répété plus il est dépourvu d'intention psychologique. L'obtention de l'auto-mouvement invite à la spontanéité intellectuelle et nous fait découvrir progressivement l'essence même du sujet.

Le cinématographe, écriture neuve, devient méthode de découverte, cela parce qu'une mécanique fait surgir l'inconnus.

20 Le ton lent et laconique de la voix des modèles est très caractéristique de cette volonté cinématographique de dépouillement sémiologique. Les dialogues sont dits plus qu'ils ne sont joués. La voix ne laisse transparaître aucune émotion; elle n'adopte aucune expressivité convenue. Bresson dénie toute tonalité injonctive de telle sorte que le spectateur ait pour fond sonore une polyphonie monocorde. Le code verbal est refoulé, symptôme de «la dislocation du monologue intérieur ". Pourtant les mots surgissent peu à peu, ils résonnent entre eux et se font écho. Cette voix douce, sans modulation rythmique et tonale, nous livre une parole réticente, timide et mystérieuse.

Modèles. Sa voix (non travaillée) nous donne son caractère intime et sa philosophie mieux que son aspect physique ${ }^{10}$.

21 Pas de psychologie ni de rôle de composition pour le modèle car Bresson exulte tout jeu mimétique. Les modèles ne sont pas le simulacre du réel car ils sont le réel. Ils doivent être " capables de se soustraire à leur propre surveillance, capables d'être divinement soi »" ${ }^{11}$. Les modèles incarnent la figure du funambule qui, en fermant les yeux, se laisse guider par son inconscience - exposition d'une conscience refoulée. L'inconscient est cet état inaccessible de la conscience qui se trahit parfois, pendant les rêves et les actes manqués pour Freud, au moyen de la répétition de gestes pour Bresson. Le cinéaste rejoint ainsi les mécanismes inconscients de la pensée, cherchant à dévoiler avec pudeur les secrets intimes de chaque individu. Si le spectateur ne se reconnait pas dans le comportement de ces modèles, en revanche il ne peut qu'adhérer à leur entreprise métaphysique. L'inaccessible devient visible.

22 Le corps brisé du curé d'Ambricourt, dans Journal d'un curé de campagne, aussi bien affaibli par sa maladie qu'impuissant face au doute sacerdotal qui l'envahit. 


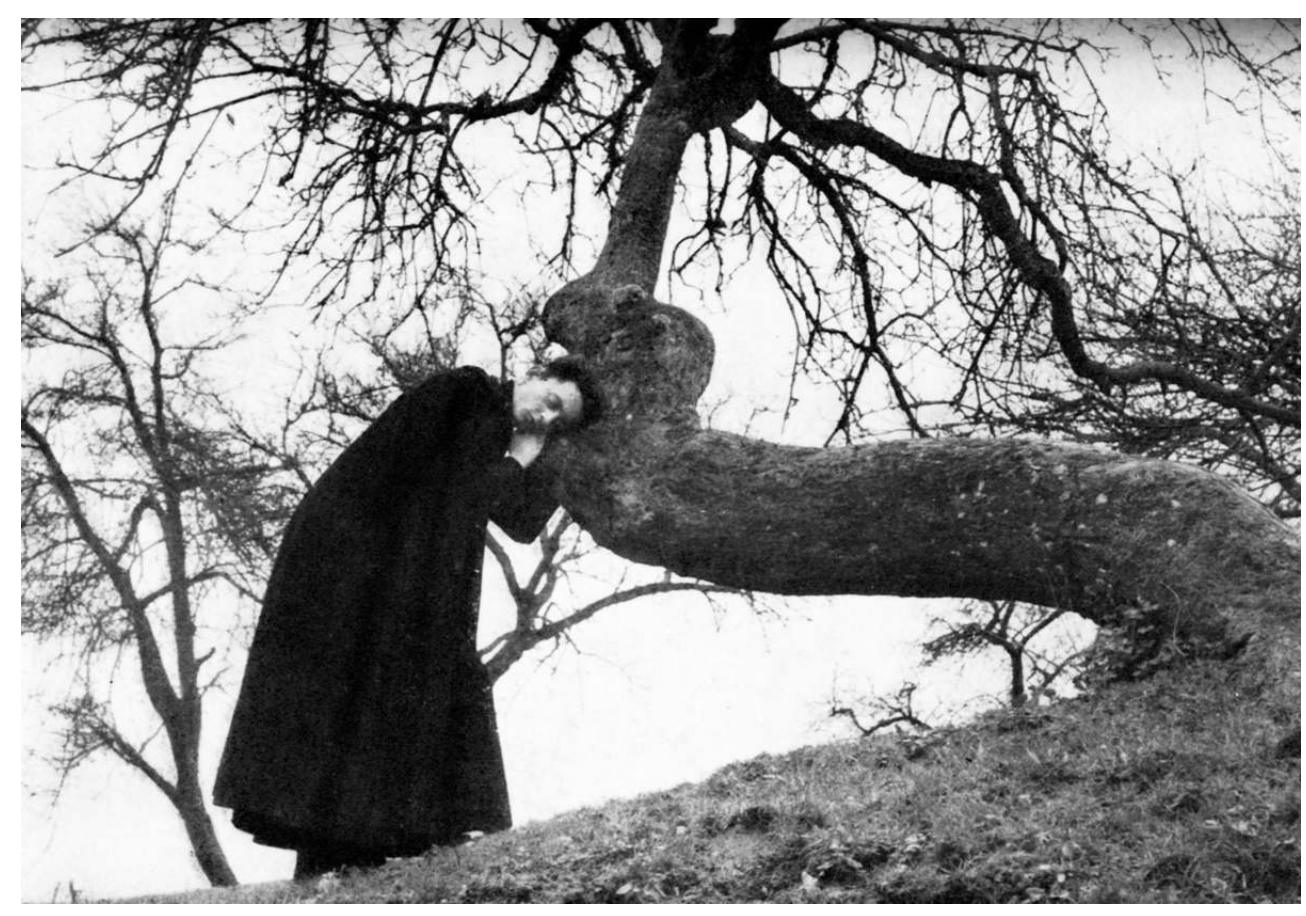

Le mécanisme du corps dévoile les mécanismes de la pensée dans un spectacle sonore et visuel où la compréhension et le devenir des âmes sont au cœur de la démarche esthétique du cinéaste.

Robert Bresson use d'une technique cinématographique capable de transformer et de dépasser le réel, de forcer la pensée à penser en mettant du sens entre les images c'est-àdire à l'intérieur du non-mouvement. Ce qui invite à penser c'est l'impensé, le non-sens ; ce lieu du non-sens est le fragmentaire, la pensée morcelée, la vie primitive, les affects, le corps. L'évolution artistique bressonienne va du percept au concept: le corps devient outil de la pensée, c'est pourquoi il est l'élément cinématographique premier, synecdoque de l'essence individuelle.

Au-delà de cette incursion existentiale, Bresson propose une véritable réflexion sur la destinée humaine ; l'écran est le miroir de la réalité des spectateurs, les hommes sont face à eux-même, face à leur "devoir-être-au-monde». Pas d'éthique, seulement la représentation de l'impuissance des hommes empreints aux passions.

\section{NOTES}

1. Cinéma II Image-temps, G. Deleuze.

2. Robert Bresson oppose le cinématographe qui se sert de la caméra comme moyen de création artistique au "théâtre filmé" qui, lui, n'est que la reproduction du réel.

3. Notes sur le Cinématographe, Bresson p. 93-94.

4. Cinéma II L'image-temps, Deleuze p. 215.

5. Terminologie bressonienne qui désigne l'acteur de cinéma.

6. Du grec automatos, qui se meut lui-même. 
7. Cinéma II L'image-temps, Deleuze p. 233.

8. Notes sur le Cinématographe, Bresson p. 70.

9. Cinéma II Image-temps, Deleuze p. 266.

10. Notes sur le Cinématographe, Bresson p. 76.

11. Notes sur le Cinématographe, Bresson p. 77.

\section{AUTEUR}

\section{PEGGY SAULE}

Doctorante, chercheur au Lara 